\title{
STUDY ON PNEUMATICS RESPONSES OF RC CIRCUITS ON VACUUM CONDITION
}

\author{
Nobuaki YAMAMOTO*, Toshinori FUJITA**, Kenji KAWASHIMA*** and Toshiharu KAGAWA*** \\ *Department of Computational Intelligence and Systems Science, Tokyo Institute of Technology \\ 4259 Nagatsuda-chou, Midori-ku, Yokohama-shi, Kanagawa-prefecture, 226-0026 Japan \\ (E-mail: yamamoto@k-k.pi.titech.ac.jp) \\ **School of Engineering, Tokyo Denki University \\ 2-2 Kandanishiki-chou, Chiyoda-ku, Tokyo, 101-8457 Japan \\ ***Precision and Intelligence Laboratory, Tokyo Institute of Technology
}

\begin{abstract}
Vacuum is frequently utilized for handling systems in factory automation. Because of the negative pressure, the state change of air is different from that of positive pressure systems during charge or discharge. However, pressure and temperature responses in the vacuum condition have not been examined up to now. Therefore, in this study the pressure and temperature responses of RC circuit in the vacuum condition is investigated.

As a result, the research can be summarized as follows:

1) When the positive and negative pressure are compared in the same pressure ratio, it is found that the heat transfer rate is larger in the positive pressure case, because of the larger mixing effect.

2) The temperature responses are determined by the thermal-time constants. Since the negative pressure gets smaller thermal-time constants which concerned the air density, the temperature responses show a smaller variation than those of positive pressure. Therefore, the pressure response is slower in the case of negative pressure.
\end{abstract}

\section{KEY WORDS}

Pneumatics Pressure, Vacuum, RC Circuits, Heat transfer

\section{NOMENCLATURE}

$C v$ : Specific heat at constant volume

$G \quad$ : Air mass flow

$h \quad:$ Heat transfer coefficient

$K_{a} \quad$ : Dimensionless number

$P \quad$ : Pressure

$P_{s} \quad:$ Supply pressure

$R \quad$ : Gus constant

Re : Reynold's number

Se : Effective area of restriction

$$
\begin{array}{lll}
S_{h} & : \text { Heat transfer area } & {\left[\mathrm{m}^{2}\right]} \\
t & : \text { Time } & {[\mathrm{s}]} \\
T_{h} & : \text { Thermal-time constants } & {[\mathrm{s}]} \\
T_{p} & : \text { Pressure-time constants } & {[\mathrm{s}]} \\
V & : \text { Chamber volume } & {\left[\mathrm{m}^{3}\right]} \\
\kappa & : \text { Specific heat ratio } & \\
\theta & : \text { Temperature } & {[\mathrm{K}]} \\
\bar{\theta} & : \text { Average temperature } & {[\mathrm{K}]}
\end{array}
$$

$[\mathrm{Pa}] \quad \bar{\theta}:$ Average temperature $\quad[\mathrm{K}]$

$[\mathrm{J} /(\mathrm{kgK})] \quad$ Subscripts
$a:$ Atmosphere $\quad u \quad:$ Charge side

$\left[\mathrm{m}^{2}\right] \quad d \quad:$ Discharge side $\quad * \quad$ : Dimensionless variable 


\section{INTRODUCTION}

At present, pneumatic devices are widely used in the industry field. Pressure levels of the pneumatic devices are not only beyond the atmosphere but also under the atmosphere. Especially, the devices operating at a low vacuum and a middle vacuum are indispensable. For example, the vacuum technology using an adsorption pad is used in the product line of semiconductor industries for the movement of products. In particular the carriage equipments need the improvements in dynamic responses and miniaturization.

Until now, many considerations of the state change of the air in positive pressure condition are done [1][2]. However, it was yet to be done at the pressure and temperature responses in the vacuum condition.

We consider the case of charge or discharge from the chamber with constant volume regardless of the internal pressure. The pressure responses in this model at this time are on the basis of the dynamic responses in pneumatics.

In this model, the pressure responses have the relation with temperature responses in the chamber. i.e., the pressure response which has fixed capacity in charge or discharge is fast in an adiabatic change, and slow in an isothermal change. However, in practice it becomes between these conditions. Therefore the pressure responses are obtained by considering the heat transfer rate between the air and the inner wall of the chamber.

In this study, this analytical model was applied to the vacuum condition, and the pressure and temperature responses of $\mathrm{RC}$ circuits in the vacuum condition are investigated. Then, the heat transfer rate was calculated using the experimental results, and compared with that in the case of positive pressure.

\section{PRESSURE RESPONSES OF RC CIRCUITS}

Fig.1 shows a basic RC circuit. When the air is charged or discharged in the chamber, the pressure response is determined by Flow Equation, State Equation and Energy Equation. These equations are shown a dimensionless variable in the following.

The pressure in the chamber and atmospheric pressure are divided by the supply pressure.

$$
\begin{aligned}
& P^{*}=\frac{P}{P_{s}} \\
& P_{a}{ }^{*}=\frac{P_{a}}{P_{s}}
\end{aligned}
$$

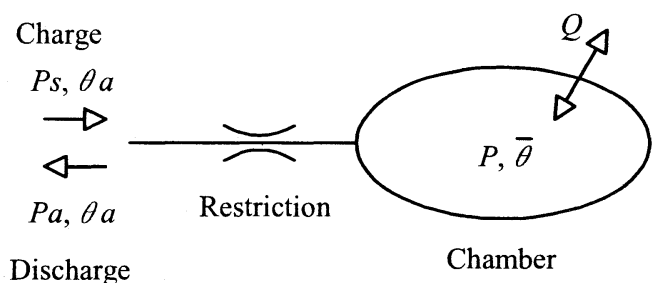

Fig.1 Pneumatic RC circuit

The dimensionless temperature is shown by using the atmospheric temperature as follows.

$$
\theta^{*}=\frac{\bar{\theta}}{\theta_{a}}
$$

\section{[Flow Equation]}

For the charge side, the flow equation is divided into two equations according to the value $P^{*}$.

The case of $P^{*} \geq 0.528$ :

$$
G_{u}^{*}=\left(\frac{\kappa+1}{2}\right)^{\frac{1}{\kappa-1}} \sqrt{\frac{\kappa+1}{\kappa-1}\left(P^{* \frac{2}{\kappa}}-P^{* \frac{\kappa+1}{\kappa}}\right)} \text {. }
$$

The case of $P^{*}<0.528$ :

$G_{u}{ }^{*}=1$.

For the discharge side, it becomes the next equation.

The case of $P_{a}{ }^{*} / P^{*} \geq 0.528$ :

$$
G_{d}^{*}=P^{*}\left(\frac{\kappa+1}{2}\right)^{\frac{1}{\kappa-1}} \sqrt{\frac{\kappa+1}{(\kappa-1) \theta^{*}\left(\left(\frac{P_{a}^{*}}{P^{*}}\right)^{\frac{2}{\kappa}}-\left(\frac{P_{a}^{*}}{P^{*}}\right)^{\frac{\kappa+1}{\kappa}}\right)}}
$$

The case of $P_{a}^{*} / P^{*}<0.528$

$$
G_{d}^{*}=\frac{P^{*}}{\sqrt{\theta^{*}}} \text {. }
$$

[State Equation of Ideal Gases]

$$
\begin{aligned}
\frac{d P^{*}}{d t^{*}} & =\theta^{*} G_{u}{ }^{*}+\frac{P d \theta^{*}}{\theta^{*} d t^{*}} \\
& =\kappa G_{u}{ }^{*}-K_{a}\left(\theta^{*}-1\right)
\end{aligned}
$$

[Energy Equation]

The energy equation in the case of charge becomes:

$$
\frac{d \theta^{*}}{d t^{*}}=\left(\kappa-\theta^{*}\right) \frac{\theta^{*} G_{u}^{*}}{P^{*}}-K_{a}\left(\theta^{*}-1\right) \frac{\theta^{*}}{P^{*}} \text {. }
$$

And it becomes the next equation in the case of discharge:

$$
\frac{d \theta^{*}}{d t^{*}}=(\kappa-1) \frac{\theta^{*^{2}} G_{d}^{*}}{P^{*}}-K_{a}\left(\theta^{*}-1\right) \frac{\theta^{*}}{P^{*}} \text {. }
$$

In the equation $K_{a}$ is dimensionless variable and it defines with the next equation:

$$
K_{a}=\frac{T_{p}}{T_{h}} \text {. }
$$

$T_{p}$ is pressure-time constant and it defines with the next equation. 


$$
T_{p}=\frac{V}{S_{e}}\left(\frac{\kappa+1}{2}\right)^{\frac{1}{\kappa-1}} \sqrt{\frac{(\kappa+1)}{2 \kappa R \theta_{a}}}
$$

The dimensionless time is shown by using $T_{p}$ as follows.

$$
t^{*}=\frac{t}{T_{P}}
$$

$T_{h}$ is thermal-time constant, which becomes the following equation.

$$
T_{h}=\frac{C v W}{S_{h} h}
$$

The velocity of the pressure response is controlled by these values. It becomes closer to isothermal condition when the value of $K_{a}$ becomes larger.

\section{EXPERIMENTAL APPARATUS AND METHODS}

\section{Experimental apparatus}

The diameter of the chamber used for the experimental apparatus is $80[\mathrm{~mm}]$, and the length is $125[\mathrm{~mm}]$. A solenoid valve and restriction are connected to this chamber. The connection tube that connected these parts was shortened as much as possible not to become flowing resistance. The pressure data was measured and stored to a computer by $\mathrm{A} / \mathrm{D}$ converter and the computer was also controlled the solenoid valve open/close state. And the sampling time of the experiment is 5 [ms].

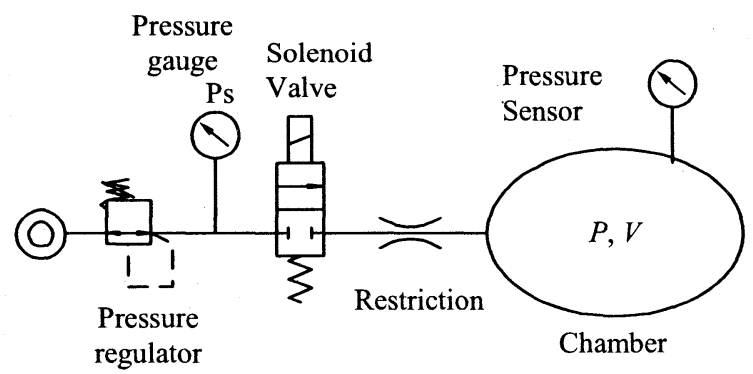

(a) The case of charge

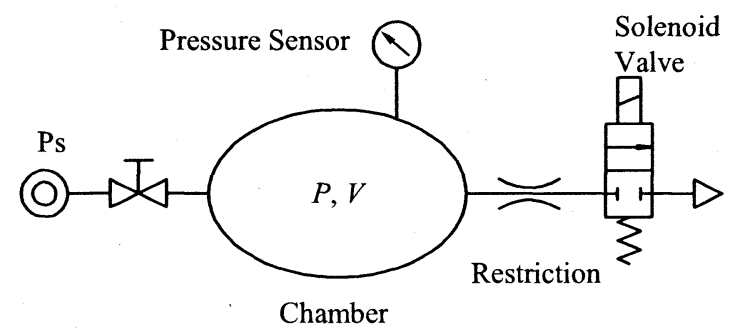

(b) The case of discharge

Fig.2 Experimental apparatus for the positive pressure measurement
Experimental apparatus for the positive pressure measurement

Fig.2 shows the experimental apparatus for the positive pressure measurement, the upper figure shows the case of charge, and the lower figure shows the case of discharge. The systems are consists of a pressure regulator, a pressure sensor, a solenoid valve, a restriction, a chamber and a pressure gauge. When it is charged, a surge tank was set up just before the solenoid valve to keep the supply pressure in the constant. When it is discharged, firstly, internal pressure of the chamber is made the supply pressure. Next, it was left until temperature became room temperature in the chamber. After that, an experiment was started.

\section{Experimental apparatus for the negative pressure measurement}

Fig.3 shows the experimental apparatus of the negative pressure measurement. The upper figure shows the case of charge and the lower figure shows the case of discharge. An ejector was used to make negative pressure occur. When it is charged, internal pressure of chamber is made vacuum condition of constant value at first. Next, it was left until

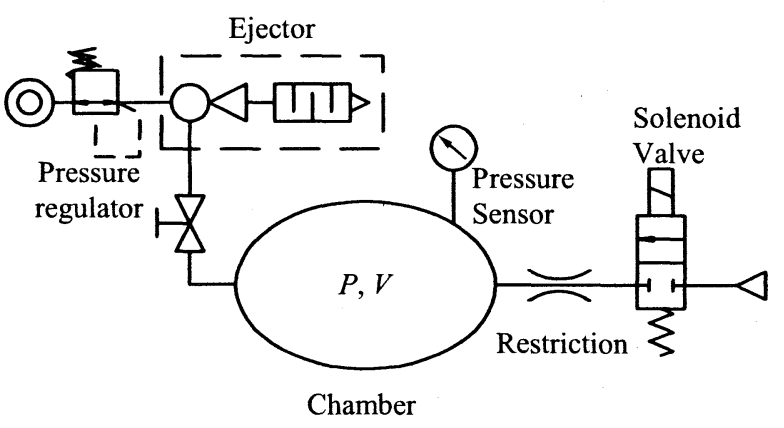

(a) The case of charge

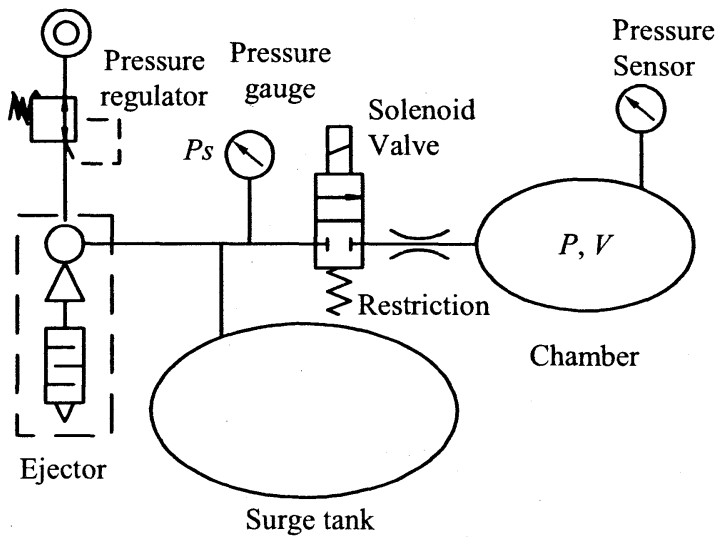

(a) The case of discharge

Fig.3 Experimental apparatus for the negative pressure measurement 


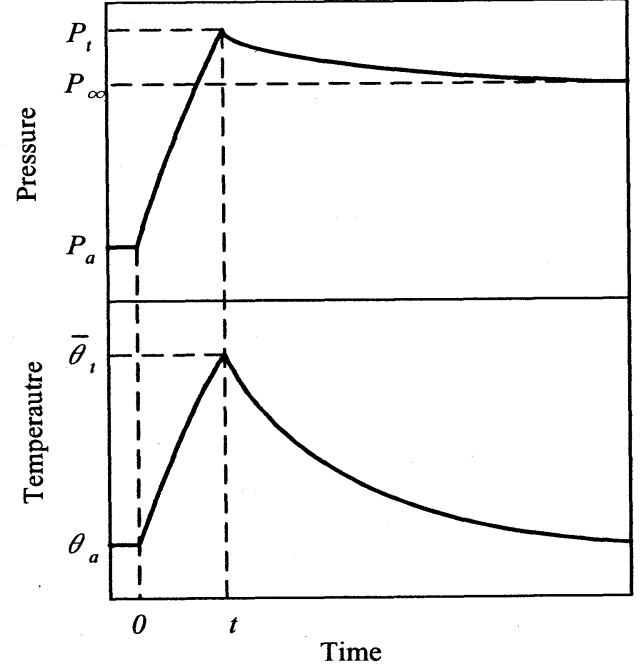

Fig.4 Pressure and temperature response in Chamber

temperature became room temperature in the chamber. After that, an experiment was started. When it is discharged, a surge tank was installed between solenoid valve and ejector so that negative pressure was not allowed to change. The surge tank used in this experiment is larger than the chamber. An experiment was started after it checked whether vacuum pressure didn't change.

\section{EXPERIMENTAL METHOD}

Fig.4 shows an example of measured pressure response curve and temperature response curve in the case of charge. The average temperature during charge or discharge was measured by using Stop Method [3] and it is explained in the following.

Measure the pressure at the time $P(t)$ and the pressure when it becomes stable $P_{\infty}$ using a pressure gauge. When the pressure becomes stable, the temperature in the chamber recovers to the room temperature. Hence, we can measure the average temperature at the time $t$ using the Law of Charles:

$$
\bar{\theta}(t)=\frac{P(t)}{P_{\infty}} \theta_{a} .
$$

By changing the time to stop charge or discharge, the average temperature at any time could be measured.

\section{EXPERIMENTAL RESULTS}

When charge or discharge is done by the same valve and pressure ratio, it can obtain the same pressure response when the isothermal change or the adiabatic change. But, when

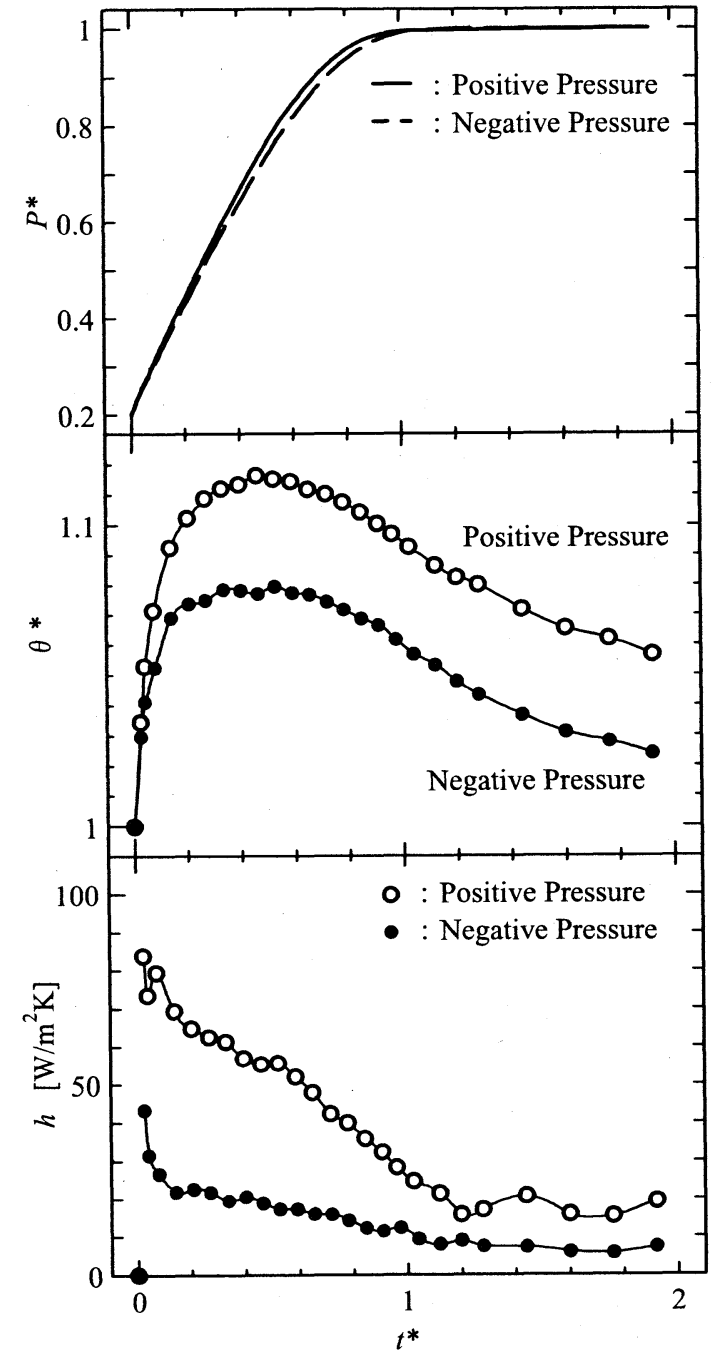

Fig.5 Experimental results of the case of charge

temperature response is considered, it becomes small when pressure level is lower. Therefore, the pressure response is slower in the case of negative pressure. These results are shown in Fig.5 and Fig.6.

Fig. 5 shows the pressure curves, temperature curves and thermal-time constants curves of the experimental results in the case of charge. The upper figure shows the pressure curves, the middle figure shows the average temperature curves and the lower figure shows the thermal-time constants curves. Pressure, average temperature and time are shown by the dimensionless variable.

As mentioned previously, the pressure response of the negative pressure condition becomes slower than positive pressure condition. Moreover, the amount of average temperature in the case of negative pressure condition becomes smaller than the positive pressure condition. The 
maximum difference is about $31 \%$.

Regarding the heat transfer rate, even though the temperature change is large at positive pressure condition, the heat transfer rate becomes 3 to 4 times larger than negative pressure.

Fig.6 shows experimental results of the case of discharge. Pressure response at discharge shows same tendency as charge. Negative pressure response becomes slower than positive pressure case. In the same way, the change of temperature was small in the negative pressure condition. The maximum difference is about $34 \%$. In next section, these reasons will be discussed.

\section{DISCUSSION}

\section{Discussion concerning heat transfer rate}

When we consider about mass flow rate of intemal chamber on charge or discharge, the average Reynold's number becomes larger as the mass flow rate becomes larger.

When the case of positive and negative pressure condition is compared, to realize the same pressure response change, the average Reynold's number of positive pressure condition becomes larger, because the mass flow rate becomes larger at positive pressure condition than negative pressure condition.

Fig.7 shows that results. This figure shows the average Reynold's number, and upper figure shows the case of charge, lower figure shows the case of discharge. The lateral axis shows the dimensionless time.

As it can be seen in experiment, the Reynold's numbers are found to be 5 times larger at the maximum in the positive pressure condition both at charge and discharge.

Generally the heat transfer rate is controlled by the Reynold's number and the Prandtle number. The heat transfer rate becomes large as much as the Reynold's number becomes large, because effect to stir is big inside the chamber. This is the reason the heat transfer rate becomes larger at the positive pressure condition.

\section{Discussion concerning thermal-time constants}

The temperature response of $\mathrm{RC}$ Circuit is determined by the dimensionless variable $K_{a} . K_{a}$ is shown in equation (8). It is described by the ratio of dimensionless number, which are pressure-time constants and thermal-time constants. The pressure-time constants $T_{p}$ doesn't depend on pressure, and it is determined by the effective area of restriction and volume of chamber. In other words, it doesn't change by the pressure

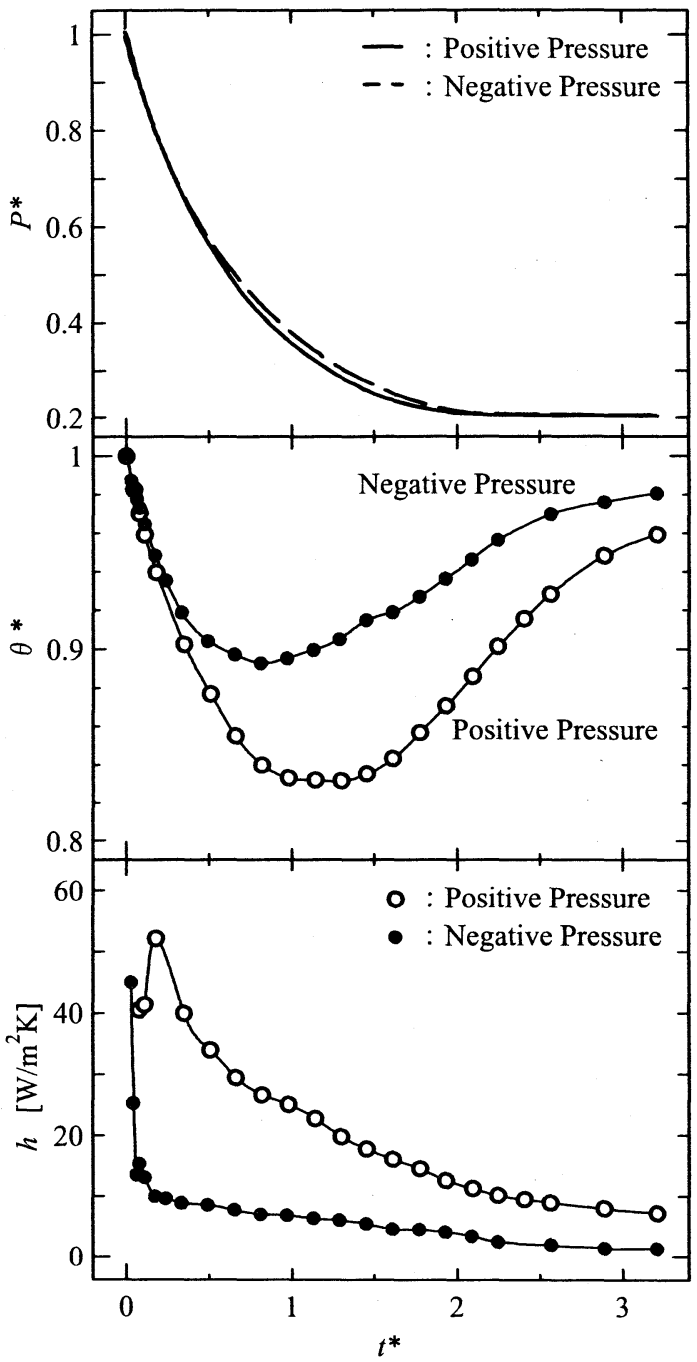

Fig.6 Experimental results of the case of discharge condition

On the other hand, the thermal-time constants becomes 20 to $30 \%$ smaller value at negative pressure condition than at positive pressure condition. The reason is even the heat transfer rate becomes 3 to 4 times larger, because of the reason discussed above, the density becomes about 5 times larger at positive pressure condition compared with negative pressure condition. That result is shown in Fig.8.

The difference of temperature response is small until about $t^{*}=0.2$ after an experiment starts, because there is no difference at thermal-time constants.

After $t^{*}=0.2$, as the thermal-time constants becomes larger at positive pressure condition than negative pressure condition, the temperature responses become different. As a result, it becomes closer to isothermal change as $K_{a}$, which determines the temperature change, becomes larger. Hence, 


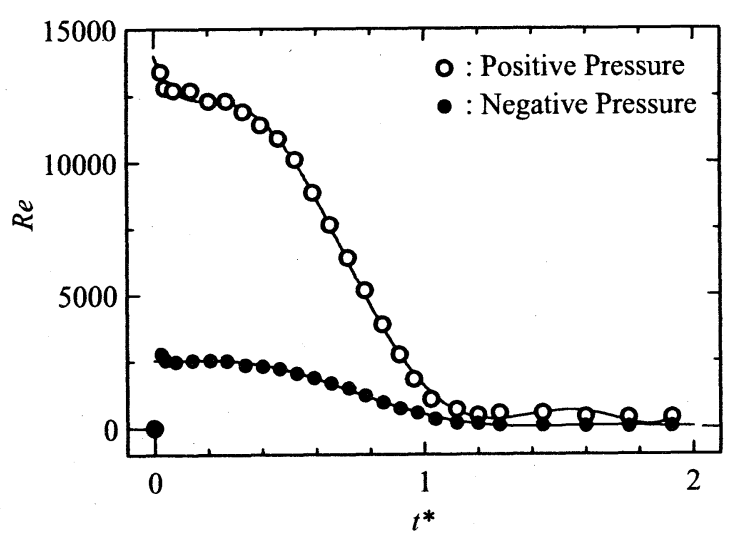

(a) The case of charge

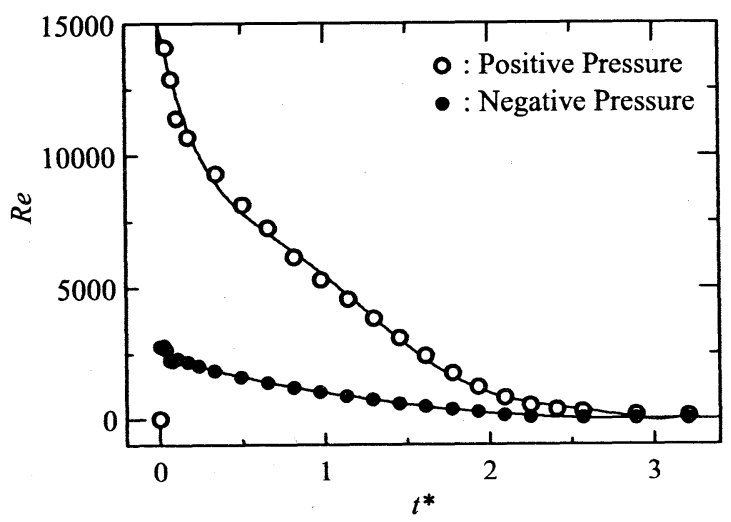

(b) The case of discharge

Fig.7 Average Reynold's Number

it is closer to isothermal condition at negative pressure. Therefore, The pressure response is slower in the case of vacuum condition.

\section{CONCLUSIONS}

In this research, the analysis of positive pressure responses considering heat transfer was applied to the vacuum condition. As a result, the conclusions of this research are as follows:

(1) When the positive and negative pressure conditions are compared in the same pressure ratio, it is found that the heat transfer rate is bigger in the positive pressure case, because the density of the negative pressure condition is lower.

(2) The heat transfer rate becomes smaller as the Reynold's number becomes smaller, because the heat transfer rate is controlled by the Reynold's number and the Prandtle number.

(3) The temperature responses are determined by the thermal-time constants. Since the negative pressure case gets smaller thermal-time constants, which concerned the air

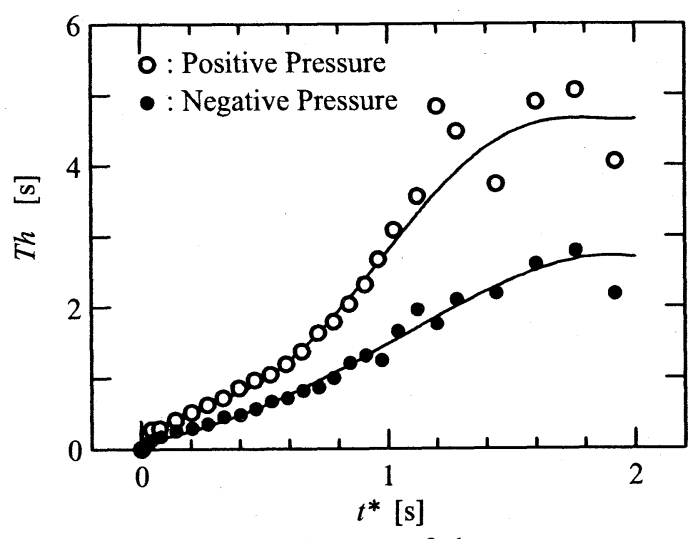

(a) The case of charge

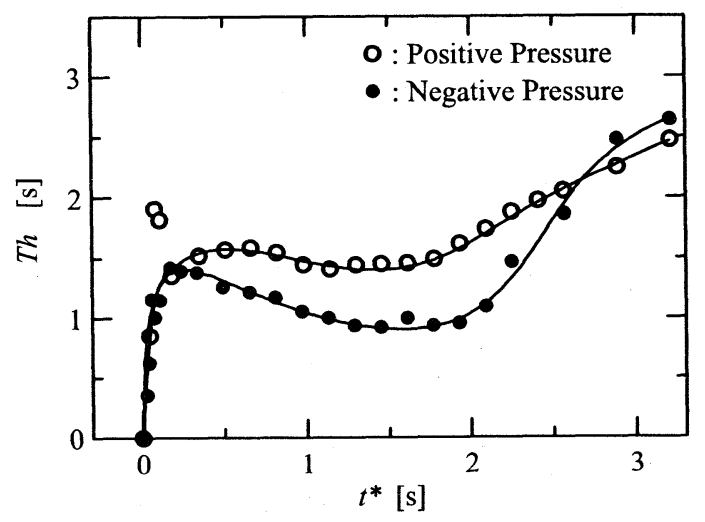

(b) The case of discharge

Fig. 8 Thermal-time constant

density, the temperature responses show a smaller variation than those of positive pressure case, Therefore, the pressure response is slower in the case of negative pressure.

\section{REFERENCES}

[1] T. Kagawa, Heat Transfer Effects on the Frequency Response of a Pneumatic Nozzle Flapper, ASME J. Dyn. Syst., Meas., Control, 1985, 107, pp.332-336.

[2] K. Kawashima, T. Fujita, T. Kagawa, Instantaneous Flow Rate Measurement of Ideal Gases, ASME J. Dyn. Syst., Meas., Control, 2000, 122, pp. 174-178,

[3] T. Kagawa and M. Shimizu, Heat Transfer Effect on the Dynamic of Pneumatic RC Circuit, 2nd International Symposium on Fluid Control Measurement and Visualization, Sheffield, England, 1988, pp. 498-502. 